\title{
Plasmapheresis: A Retrospective Audit of Procedures from a Tertiary Care Center in Southern India
}

\author{
0. T. Ahammed Nizar, Pratheeksha Rai, Shobhana Nayak Rao', M. Pradeep Shenoy ${ }^{1}$ \\ Departments of Internal Medicine and ${ }^{1}$ Nephrology, K.S. Hegde Medical Academy, Mangalore, Karnataka, India
}

\section{Abstract}

Introduction: The term plasmapheresis/plasma exchange refers to the removal of the plasma component of blood and its replacement with various fluids. Plasma Exchange (PE) has been used to treat a variety of conditions that are associated with an aberrant immune response. We undertook this retrospective study aiming to look at plasmapheresis procedures conducted in the nephrology department over a fixed time period. Materials and Methods: Retrospective analysis of PE procedures from January 2013 to October 2016 was conducted in the nephrology and Intensive Care Unit of a tertiary care teaching hospital. The goal was to achieve a total removal of 150-200 ml/plasma per $\mathrm{kg}$ body weight. As replacement, we used a standard protocol of $100 \mathrm{ml}$ of $20 \%$ albumin in $1 \mathrm{~L}$ of normal saline and $2-3$ units of fresh frozen plasma. All results were expressed as mean \pm standard deviation and statistical analysis was done using the Student's $t$-test for continuous and Fisher's exact test for categorical data. Results: A total of 192 procedures performed on 40 patients (22 males and 18 females). Age ranged from 15 to 79 years with a mean age of 37.5 years. Guillain-Barre syndrome accounted for 67.5\% (>two-third of causes) for PE. Vascular access was femoral catheter in 27 (67.5\%) and internal jugular catheter in $13(32.5 \%)$. Mild hypotension occurred in 15 procedures (7.8\%) of patients and allergic reactions such as rashes and chills occurred in 5 cycles $(2.6 \%)$. A total of 36 patients $(90 \%)$ showed significant improvement in condition, 2 did not show any change, while one worsened and one died due to respiratory complications. Conclusion: Our small series of data of plasmapheresis procedures from nephrology perspective has reaffirmed the safety and efficacy of the therapy in an experienced setup.

Keywords: Complications, dialysis, fluid replacement, plasma exchange

\section{INTRODUCTION}

The term "apheresis" has been used to describe the process of removal of blood constituents by extracorporeal blood purification methods. The term plasmapheresis refers to the removal of the plasma component of blood and plasma exchange (PE) which involves separation and removal of plasma, and its replacement with various fluids is used interchangeably and synonymously. Plasmapheresis (PE) has been used to treat a variety of conditions that are associated with an aberrant immune response. It was first used in 1952 in the setting of multiple myeloma to treat hyperviscosity ${ }^{[1]}$ and since then has emerged as an important treatment modality for a number of neurological and other conditions. ${ }^{[2]}$ Indication guidelines have been defined and revised in 2010 by the American Society for Apheresis and divided into four categories from 1 to 4 on the basis of available literature. ${ }^{[3]}$ Category 1 disorders are those for which plasmapheresis is accepted as first-line therapy either as primary standalone

\begin{tabular}{|l|l|}
\hline \multicolumn{3}{|c|}{ Access this article online } \\
\hline Quick Response Code: & Website: \\
\hline & www.ijccm.org \\
\cline { 2 - 2 } & \\
\hline
\end{tabular}

treatment or in conjunction with other models of treatment and include disorders such as Guillain-Barre syndrome (GBS), myasthenia gravis, chronic inflammatory demyelinating polyneuropathy, thrombotic thrombocytopenic purpura, Goodpasture's syndrome, and atypical hemolytic uremic syndrome. The separation of plasma from blood can be achieved by centrifugation devices or with the use of hemodialysis machine and plasma filters. Although an Indian Society for Apheresis was created in 1985 , there is a scarcity of data on PE from the Indian subcontinent. This is partly because the facility for PE is available in only large centers located mainly in the cities. With the aim of improving data collection about plasmapheresis procedures in the country, we undertook

Address for correspondence: Dr. Shobhana Nayak Rao,
Department of Nephrology, K.S. Hegde Medical Academy,
Mangalore, Karnataka, India.
E-mail: nayak_shobhana@rediffmail.com

This is an open access article distributed under the terms of the Creative Commons Attribution-NonCommercial-ShareAlike 3.0 License, which allows others to remix, tweak, and build upon the work non-commercially, as long as the author is credited and the new creations are licensed under the identical terms.

For reprints contact: reprints@medknow.com

How to cite this article: Ahammed Nizar OT, Rai P, Rao SN, Shenoy MP. Plasmapheresis: A retrospective audit of procedures from a tertiary care center in Southern India. Indian J Crit Care Med 2017;21:857-60. 
this retrospective study aiming to look at plasmapheresis procedures conducted in the nephrology department over a fixed time period.

The procedure for plasma exchange involves removing $30-40 \mathrm{ml} / \mathrm{kg}$, or $1.5-2 \mathrm{~L}$ of plasma is removed at each session for a total of 5-7 sessions to reach a therapeutic target of 150-200 ml of plasma removed per kg body weight. Replacement involves use of albumin, fresh frozen plasma (FFP) or both, and saline. There are no fixed guidelines on the ideal replacement solution that needs to be used and most centers follow their own replacement protocols. To reduce the risk of complications, the use of albumin and cross-matching of plasma has been recommended. ${ }^{[4]}$ The efficacy of therapeutic PE has been thought to be due to a variety of possible mechanisms which include removal of antibody, immune complexes, monoclonal protein, toxins or cytokines, and replacement of a specific plasma factor. ${ }^{[5,6]}$ Plasmapheresis has been documented to be an extremely safe procedure in experienced hands. Common complications such as hypotension, fluid-electrolyte imbalance, fever, and chills have been reported; however, they are treatable immediately and not life-threatening and are rarely serious enough to discontinue therapy. ${ }^{[7]}$ Other serious complications such as those related to the vascular access such as pneumothorax, thrombosis, and infection, bleeding due to anticoagulant use, and increased risk of infection are rare. ${ }^{[8]} \mathrm{We}$ analyzed the plasmapheresis procedures done at the nephrology department at our institute and reported our findings.

\section{Materials and Methods}

We retrospectively reviewed all PE procedures during a period of 46 months from January 2013 to October 2016 in the nephrology department including the dialysis unit and Intensive Care Unit of a tertiary care teaching hospital. The diagnosis and indication for plasmapheresis were established by proper clinical and laboratory evaluation. The patient's age, weight, height, gender, and clinical indication along with preprocedure hematological parameters (complete blood count, bleeding, clotting time), renal, liver function tests and serology for HIV, hepatitis B and C viruses were recorded. A baseline chest X-ray and electrocardiogram were also done before the procedure. All procedures were done by experienced dialysis personnel under the supervision of medical resident who reported to the treating nephrologist. A repeat chest X-ray was done to confirm catheter position after insertion of an internal jugular catheter. The goal was to perform five exchanges with $30-50 \mathrm{ml} / \mathrm{kg}$ of plasma removal per session to achieve a total removal of $150-200 \mathrm{ml} /$ plasma per $\mathrm{kg}$ body weight. Heparin was given in a bolus dose of 1000 units in the beginning every $30 \mathrm{~min}$ to prevent clotting in the extracorporeal circuit. Once the target plasma ultrafiltrate was obtained, the procedure was discontinued. As replacement, we used a standard institution-based protocol of $100 \mathrm{ml}$ of $20 \%$ albumin diluted in $1 \mathrm{~L}$ of normal saline along with 2-3 units of FFP which were infused at the end of the procedure. All PE procedures were done in the renal or neurological Intensive Care Units using the Fresenius Medical Care Dialysis machine (4008S) by the continuous flow method with the Plasmaflux PSU 2S (Fresenius Medical Care). All results were expressed as mean \pm standard deviation and statistical analysis was done using the Student's $t$-test for continuous data and Fisher's exact test for categorical data. The hospital Institutional Ethics Committee approved the study.

\section{RESULTS}

A total of 192 procedures were performed on 40 patients. There were 22 males and 18 females. Age ranged from 15 to 79 years with a mean age of 37.5 years. Indication for which plasmapheresis was done are given in Table 1. GBS accounted for $67.5 \%$ (>two-third of causes) for PE with neuromyelitis optica being the second common cause in $10 \%$ of patients. Previous co-morbid medical conditions included diabetes mellitus in $8(20 \%)$, hypertension in 7 (17.5\%), chronic obstructive pulmonary disease in $2(5 \%)$ patients, and ischemic heart disease in $2(5 \%)$ patients. All patients were medically stable at the time of PE. Vascular access was femoral catheter in 27 (67.5\%) of patients while internal jugular catheter was used in $13(32.5 \%)$ of patients. We did not have any immediate complications related to catheter placement since all catheters are inserted under ultrasound guidance. In patients with GBS, PE was done as first-line therapy in $>90 \%$ of patients. None of our patients was pregnant at the time of PE.

Although PE protocol is for an average of 5 exchanges, it was extended to 7 cycles in 3 patients [Table 2]. Twenty-six (65\%) of patients however received 5 cycles of treatment. Eight patients had $<5$ cycles; these were mainly related to issues such as filter clotting and/or significant improvement after fewer exchanges. No patient required discontinuation of treatment due to side effects.

With regard to side effects of procedure, mild hypotension occurred in 15 procedures $(7.8 \%)$ of patients and this was

\begin{tabular}{lc}
\hline Table 1: Indications for plasmapheresis & \\
\hline Indication & $\boldsymbol{n}(\%)$ \\
\hline GB syndrome & $27(67.5)$ \\
Myasthenia gravis & $3(7.5)$ \\
Neuromyelitis optica & $4(10)$ \\
Anti-GBM disease & $1(2.5)$ \\
Others & $5(8)$ \\
\hline GBM: Glomerular basement membrane; GB: Guillain-Barre &
\end{tabular}

Table 2: Total number of procedures performed

\begin{tabular}{lc} 
Number of cycles & Patient number \\
\hline 7 cycles & 3 \\
6 cycles & 2 \\
5 cycles & 26 \\
4 cycles & 6 \\
3 cycles & 1 \\
2 cycles & 2 \\
\hline
\end{tabular}


fully reversible with discontinuation of procedure and/or saline infusion. Allergic reactions such as rashes and chills occurred in 5 cycles $(2.6 \%)$ and these too rapidly responded to symptomatic therapy. As mentioned earlier, our center has a standard policy of ultrasound-guided catheter insertion, and this may have accounted for nil vascular access-related complications.

Thirty-six patients (90\%) showed significant improvement in condition after PE, 2 patients did not show any change, while one patient worsened and one patient died due to respiratory infection-related complications. There was no procedure-related mortality.

\section{Discussion}

We report a series of 40 patients who underwent 192 cycles of PE over a 46-month period for various indications. The major indication for PE in our study was neurological of which GBS accounted for $67.5 \%$ of patients undergoing PE for this condition. In GBS, the recommended treatment options of PE or intravenous IgG (IVIg) have both been found to be equally effective. ${ }^{\left[{ }^{9,10]}\right.}$ Initially, IVIg was preferred due to the ease of administration and familiarity of use. However, PE has proven to be more cost-effective at least in the Indian scenario when compared to IVIg therapy (approximately half the cost) and with improvements in procedure safety and technique has proved to be extremely safe in experienced hands. It must be stressed that plasmapheresis was the first-line therapy in patients with neurological indications such as GBS, while in other indications, it was add-on therapy with other immunosuppressive therapy including steroids and antimetabolites such as cyclophosphamide/mycophenolate mofetil.

The majority of our patients were adults (mean age 37.5 years) with range of 15-79 years. Older age is a higher risk factor for complications due to higher chance of hemodynamic alterations with one study finding a complication risk of $11.5 \%$ in patients older than 65 years compared to $3.9 \%$ in the younger age group. ${ }^{[1]}$ This could also be due to the presence of preexisting conditions such as diabetes mellitus and ischemic heart disease. Diabetics constituted 20\% of patients in our group while cardiovascular disease was seen in 5\%. Hypotension, allergic reactions, nausea, vomiting paresthesia, and cramps are the most common complications, and these may be seen in $3 \%-25 \%$ of procedures. ${ }^{[12]}$ Most of these events are mild and resolve without treatment. The reported incidence of paresthesia and cramps ranges from $1.5 \%$ to $9 \%{ }^{[12]}$ Reported incidence of hypotension ranges from $2.6 \%$ to $8.1 \%{ }^{[13-15]}$ However, the incidence of both of these was quite low in our study. The overall mortality in PE is estimated to be $1-3 / 10,000$ procedures, ${ }^{[16]}$ and we had one mortality in our group. Previously reported data from India ${ }^{[17,18]}$ had shown higher incidence of adverse events. However, more recent data $^{[19,20]}$ from India have demonstrated the easy feasibility and low rate of complications associated with plasmapheresis techniques in well-equipped settings. It has also been shown to be more cost-effective when compared to other interventions such as IVIg after taking into account the initial cost of procurement of setup and training of staff. Complication rates and mortality did not vary significantly among the different clinical indications in our study. The limitation of our study was its retrospective nature and the fact that it was a single-center study. However, our study had a good patient number which to some extent overcomes this limitation.

In India, blood cell separators and centrifugation techniques have been commonly used to separate red blood cells and plasma, and very few superspeciality centers with well-developed dialysis facility and trained staff and personnel at present perform therapeutic apheresis. ${ }^{[13,14]}$ According to Sharma et al., ${ }^{[17]}$ there has been a 3 -fold rise in patient referrals for PE between the years 2005-2009 compared to 2000-2004. Training and expertise from apheresis experts have been shown by Tiwari et al. ${ }^{[21]}$ to improve comfort with the use of and thereby contribute to safety of technique. This has been due to evolvement of safe and efficient machines and techniques and an encouraging clinical response. There is however a relative scarcity of published data on $\mathrm{PE}$, and there is currently a need to accurately collect and send data of plasmapheresis procedures from various centers in India to the Indian apheresis registry. This would be helpful for better understanding of the PE scenario as well as newer applicability and feasibility of the procedure across various centers.

\section{ConcLusion}

Our small series of PE data from nephrological perspective has reaffirmed the safety and efficacy of the procedure in an experienced setup. It is imperative that accurate procedural data need to be sent to the national registry so that the benefit of therapy can be extended to other potential disorders.

\section{Financial support and sponsorship}

Nil.

\section{Conflicts of interest}

There are no conflicts of interest.

\section{REFERENCES}

1. Kaplan AA. Therapeutic plasma exchange: Core curriculum 2008. Am J Kidney Dis 2008;52:1180-96.

2. Strauss RG, Ciavarella D, Gilcher RO, Kasprisin DO, Kiprov DD, Klein HG, et al. An overview of current management. J Clin Apher 1993;8:189-94.

3. Schwartz J, Padmanabhan A, Aqui N, Balogun RA, Connelly-Smith L, Delaney $\mathrm{M}$, et al. Guidelines on the use of therapeutic apheresis in clinical practice-evidence-based approach from the writing committee of the American Society for Apheresis: The Seventh Special Issue. J Clin Apher 2016;31:149-62.

4. Korach JM, Berger P, Giraud C, Le Perff-Desman C, Chillet P. Role of replacement fluids in the immediate complications of plasma exchange. French Registry Co-operative Group. Intensive Care Med 1998;24:452-8.

5. Goto H, Matsuo H, Nakane S, Izumoto H, Fukudome T, Kambara C, et al. Plasmapheresis affects $\mathrm{T}$ helper type- $1 / \mathrm{T}$ helper type- 2 balance of 
circulating peripheral lymphocytes. Ther Apher 2001;5:494-6.

6. Reeves JH, Butt WW, Shann F, Layton JE, Stewart A, Waring PM, et al. Continuous plasmafiltration in sepsis syndrome. Plasmafiltration in Sepsis Study Group. Crit Care Med 1999;27:2096-104.

7. Couriel D, Weinstein R. Complications of therapeutic plasma exchange: A recent assessment. J Clin Apher 1994;9:1-5.

8. Mokrzycki MH, Kaplan AA. Therapeutic plasma exchange: Complications and management. Am J Kidney Dis 1994;23:817-27.

9. van der Meché FG, Schmitz PI. A randomized trial comparing intravenous immune globulin and plasma exchange in Guillain-Barré syndrome. Dutch Guillain-Barré study group. N Engl J Med 1992;326:1123-9.

10. Cortese I, Chaudhry V, So YT, Cantor F, Cornblath DR, Rae-Grant A. Evidence - Based guidelines update. Plasmapheresis in neurologic disorders: Reports of the Therapeutics and Technology Assessment subcommittee of the American Academy of Neurology. Neurology 2011;76:294-300.

11. Basic-Jukic N, Brunetta B, Kes P. Plasma exchange in elderly patients. Ther Apher Dial 2010;14:161-5.

12. Stegmayr B, Ptak J, Wikström B, Berlin G, Axelsson CG, Griskevicius A, et al. World apheresis registry 2003-2007 data. Transfus Apher Sci 2008;39:247-54.

13. Srivastava R, Gupta RC, Khatri PC, Lalchandani A. Changing scenario of therapeutic apheresis in India in the last 14 years. Ther Apher $1998 ; 2: 317-9$
14. Vucic S, Davies L. Safety of plasmapheresis in the treatment of neurological disease. Aust N Z J Med 1998;28:301-5.

15. Shemin D, Briggs D, Greenan M. Complications of therapeutic plasma exchange: A prospective study of 1,727 procedures. J Clin Apher 2007;22:270-6.

16. Ward DM. Conventional apheresis therapies: A review. J Clin Apher 2011;26:230-8.

17. Sharma RR, Saluja K, Jain A, Dhawan HK, Thakral B, Marwaha N, et al. Scope and application of therapeutic apheresis: Experience from a tertiary care hospital in North India. Transfus Apher Sci 2011;45:239-45.

18. Gafoor VA, Jose J, Saifudheen K, Musthafa M. Plasmapheresis in neurological disorders: Experience from a tertiary care hospital in South India. Ann Indian Acad Neurol 2015;18:15-9.

19. Vikrant S, Thakur S, Sharma A, Gupta D, Sharma S. Safety and efficacy of therapeutic membrane plasmapheresis in the treatment of Guillain-Barré syndrome: A study from a tertiary care hospital from India. Neurol India 2017;65:527-31.

20. Tiwari AK, Bhardwaj G, Aggarwal G, Arora D, Dara RC, Acharya DP, et al. Changing trends in therapeutic plasmapheresis: An Indian perspective. Ther Apher Dial 2017;21:500-6.

21. Tiwari AK, Dara RC, Pandey P, Arora D, Rawat G, Raina V, et al. Change in therapeutic apheresis practices: Role of continuing medical education (CME). J Clin Apher 2016;31:16-21. 